\title{
CUMULANTS AND PARTITION LATTICES V. CALCULATING GENERALIZED $k$-STATISTICS
}

\author{
T. P. SPEED and H. L. SILCOCK \\ (Received 1 October 1985; revised 5 January 1987)
}

Communicated by T. C. Brown

\begin{abstract}
A method is developed for obtaining compact, easily computed and statistically interpretable expressions for the generalized $k$-statistics associated with multiply-indexed arrays of random variables such as those which arise in variance component analysis. These expressions will be used in the next paper in this series to give formulae for variances and covariances of estimates of components of variance.
\end{abstract}

1980 Mathematics subject classification (Amer. Math. Soc.): 62 A 05, 62 J 10.

$K e y w o r d s$ and phrases: cumulant, $k$-statistic, analysis of variance.

\section{Introduction}

In previous papers in this series, Speed (1986a,b), hereafter referred to as II, III, we gave definitions of cumulants and $k$-statistics for singly and multiply indexed arrays generalizing the classical expressions with these names, and showed how they were useful in a number of ways including proving the a.s. convergence of standard variance component estimates to their expectations under quite general conditions. The original aim in developing this machinery was to give an account of the theory of variances and covariances of estimates of components of variance under the widest assumptions possible, including the classical linear models with independent normal effects on one hand, and the randomization models of Kempthorne (1952), his co-workers and students, on the other. We refer to the introduction of III for further background comments. In this and the next paper in the series we hope to show that the above aim can be achieved in

(C) 1988 Australian Mathematical Society $0263-6115 / 88 \$ A 2.00+0.00$ 
a very satisfactory way, thereby, we hope, completing a chapter in this theory which began as far back as the mid-1950s in the work of Kempthorne, Tukey and their successors.

The present paper plays a crucial technical role in the fulfilment of our original aim, for we need compact and readily computed expressions for our generalized $k$-statistics; these are derived below. In one sense this paper is a technical interlude before we get down to the real business of computing variances and covariances of mean squares, but in another sense the results reported below lie right at the heart of the matter. Without the simplifications that are found below the general approach adopted in this series of papers would be of only limited use in achieving the original aim; with them, we have some very useful computing formulae which we hope will lead to a greater understanding of the assumptions underlying standard variance component models. In this sense we feel that the results given below are of independent interest, although further development is still necessary.

\section{The problem}

Why is there a problem at all in computing generalized $k$-statistics? To see this we should begin with a statement of why we should want to compute generalized $k$-statistics. When the order $m=2$ this is quite clear, for we have shown in III that under an additive model, generalized $k$-statistics are the symmetric (and minimum variance) quadratic unbiassed estimators of the associated variance component parameters. Indeed we have also seen in this context that for order $m>2$, they correspond to best estimators of the skewness, kurtosis, etc. parameters of the component random effects. If we want to estimate these parameters, for example, the kurtosis of the within class effect associated with an additive model in the simple nested case, we will need to evaluate a fourth order $k$-statistic. Thus the need to do these evaluations is evident.

The problems associated with these evaluations are best illustrated by an example. Suppose that our underlying poset is the 2-element chain corresponding to a simple nesting of one index in another. Referring to the end of Section 2 in III we find that the generalized $k$-statistic $k(123,123)$ in this context is defined as an alternating sum of twelve normalised monomial symmetric functions $(n)_{\varphi}^{-1} a(\varphi), \varphi \in \operatorname{Hom}(P, P(3))$, see the Appendix below. If the numbers of levels of the two factors are $n_{1}=m$ and $n_{2}=n$, and $m \leq n$, then each of these monomial symmetric functions is a sum, with inequality constraints on the six indices, of up to $O\left(n^{3}\right)$ terms. Because of these constraints we would prefer not to compute the sums directly, but rather express them in terms of the power sum symmetric functions, also called unrestricted sums by Carney (1968). Each 
of these functions involves up to $O\left(n^{3}\right)$ terms (without inequality constraints) and up to twelve are included in the expression for each monomial symmetric function. Summarising this line of reasoning, we conclude that $k(123,123)$ is expressible as a sum of up to 144 terms, each of which in turn is a sum of up to $O\left(n^{3}\right)$ terms. If our interest was in $k(1234,1234)$, we would be considering the possibility of up to 3600 terms, each of which is a sum of up to $O\left(n^{3}\right)$ terms, already a formidable computing problem. Note that this is exactly how Kendall and Stuart (1969) go about an identical task: see Exercise 12.11 on page 304. Similarly Kaplan (1952) expresses $k$-statistics in terms of power sum symmetric functions.

Is all this necessary? In III, Section 2 we found that the answer was no, for there we gave an expression for $k(123,123)$ involving sums over $i$ and $j$ once only, that is, $m n$ terms. Of course, the terms in this expression are not as simple as those involved in the power sum symmetric functions, but they have the gread advantage of being easily computed, and more importantly, interpretable statistically. Indeed, if we were to consider actual numbers rather than abstract symbols, in the context of an analysis of variance, then the components in the simple expression for $k(123,123)$, would usually be available. In any event the expression for $k(123,123)$ given in III Section 2 is a simple form, which is readily interpreted statistically, and it is the main purpose of this paper to obtain analogous expressions for all generalized $k$-statistics. We remark in passing that the simplifying which can be done quite readily by hand for $k(123,123)$ is already very difficult to do this way for $k(1234,1234)$, although the final expression for $k(1234,1234)$ involves at most four sums each of $m n$ terms, rather than the 3600 mentioned above. Our expressions are natural generalizations of expressions for $k$-statistics in terms of centred moments, see Kendall and Stuart (1969, page 281).

Two further points are worth making concerning our formulae. Firstly we remark that although they are almost certainly not the best expressions for computing with real numbers, our formulae are certainly better than the ones given in terms of a large number of alternating sums. The situation is completely analogous to (and indeed generalizes) the computation of $k(2)=n^{-1} \sum_{i} x_{i}^{2}-$ $[n(n-1)]^{-1} \sum \sum_{i \neq j} x_{i} x_{j}$. One method expresses this as the difference $\sum_{i} x_{i}^{2}-$ $n^{-1}\left(\sum_{i} x_{i}\right)^{2}$ between two sums which may be very close together whilst the other writes it as a sum of squares of $n$ differences computed first: $(n-1)^{-1} \sum_{i}\left(x_{i}-\right.$ $\bar{x})^{2}$. It is well known that the second method is far better than the first for computation although other recursive methods have proved even more stable. Whilst such methods are no doubt available for our more general expressions, we have not pursued this aspect any further.

The second point we wish to make relates to the statement on III, page 169 that "No general theory of such simplifications exists". The simplifications in 
question are those we are talking about, that is, the reduction of large numbers of alternating sums to much more compact expressions involving rather different terms. As far as we are aware, this statement is still true, although some of the simplifications which we have found by using a computer to manipulate the formulae are sufficiently dramatic that one might feel there is still hope of some general theory giving the results directly. Some further remarks are made about this point at the appropriate place below.

In closing these remarks about the problem addressed in this paper we comment that it was almost certainly this problem which prevented Carney (1965) from pushing his general theory, which was similar to but not quite the same as ours, though to a satisfactory solution of the problem of computing a wide range of variances and covariances of mean squares. A considerable proportion of his thesis was devoted to the very computational issues which we are able to circumvent as a result of the theory given below. In the last section (Section 9) of his paper on what are essentially our generalized $k$-statistics (his bipolykays) when $P$ is the poset consisting of two incomparable elements, Hooke (1956) comments on the problem of computing bipolykays of degree 4 . He hoped that a better procedure than his would be found and (without having seen his) we hope that is what we are presenting.

\section{The tensors $T(\varphi)$}

We are concerned with generalized symmetric functions involving indeterminates $x_{i_{1} i_{2}} \ldots, y_{i_{1} i_{2}} \ldots$, etc. with suffixes from a multi-indexed set $I=$ $\prod\left\{\mathbf{n}_{p}: p \in P\right\}$ whose factors are labelled by a partially-ordered set (poset) $P$ describing the nesting relationships on the subscripts $i_{1}, i_{2}, \ldots$ Here $\mathbf{n}_{p}=$ $\left\{1, \ldots, n_{p}\right\}$ where $n_{p}$ is a natural number for each $p \in P$. In particular we want to look at functions built up from expressions in which some of $i_{1}, i_{2}, \ldots$ are replaced by dots, indicating an average over the corresponding index set. As in III, whose notation and results we make use of below, we work with tensors, which can be regarded as coefficients of the symmetric functions of interest, rather than directly with expressions involving $x$ 's, $y$ 's, etc.

If we take the power sum symmetric functions $R_{\varphi}$, defined in the Appendix for $\varphi \in \operatorname{Hom}(P, P(\mathbf{m}))$, and examine the expressions that result when some of the $i_{p}$ are replaced by dots, we find that every one that is invariant under the relevant permutation group, namely $G W(I)$, can be expressed as a multiple of a power sum symmetric function $R\left(\varphi \wedge \gamma_{s}\right)$, where the $\gamma_{s}$ are certain elements of $\operatorname{Hom}(P, P(\mathbf{m}))$. These $\gamma_{s}$ are labelled by elements $s$ belonging to the poset $\operatorname{Hom}(P, B(\mathbf{m}))$ of order-preserving maps from $P$ into the power set $B(\mathbf{m})$ (all subsets of $\mathbf{m}$, regarded as a poset under inclusion). 
For fixed $\varphi$, the elements $\varphi \wedge \gamma_{s}$ form a poset under the ordering induced from that of $\operatorname{Hom}(P, P(\mathbf{m}))$. We use Möbius inversion with respect to this induced ordering to define a new class of tensors (symmetric functions) $T(\varphi)$, $\varphi \in \operatorname{Hom}(P, \mathcal{P}(\mathbf{m}))$, with the property that, for each $\varphi$, the normalized symmetric function $\bar{R}(\varphi)=\left(n^{\varphi}\right)^{-1} R_{\varphi}$ is the sum of all the tensors $T\left(\varphi \wedge \gamma_{s}\right)$ corresponding to the distinct elements of the form $\varphi \wedge \gamma_{s}$. We then derive some alternative, more explicit, expressions for the $T(\varphi)$ and in the next section relate them to the generalized $k$-statistics $F(\varphi)$, the main object of our interest. We turn now to the formal definitions and proofs.

In III the tensors $A(\varphi)$ and $R(\varphi)$ were defined in terms of the elements $\bar{h}=$ $\delta^{h(1)} \otimes \cdots \otimes \delta^{h(m)}$ where $h: \mathbf{m} \rightarrow \prod \mathbf{n}_{p}$ and $\delta^{i}=\bigotimes\left\{\delta_{p}^{i_{p}}: p \in P\right\}$, the $\delta_{p}^{i_{p}}$ being elements of the canonical basis $\left\{\delta_{p}^{1}, \ldots, \delta_{p}^{n_{p}}\right\}$ of $\mathbf{R}^{n_{p}}, p \in P$. Thus we are working in the space $\left(\otimes\left\{\mathbf{R}^{n_{p}}: p \in P\right\}\right)^{\otimes m}$ and in what follows it will be convenient to change the ordering of tensoring, writing $\bar{h}=\bigotimes \bigotimes\left\{\delta_{p}^{h(l)_{p}}: p \in P, l \in \mathbf{m}\right\}$ so that we are in effect working in $V=\bigotimes\left\{\left(\mathbf{R}^{n_{p}}\right)^{\otimes m}: p \in P\right\}$. Obviously the principal effect of this change is notational.

With these changes we may define

$$
R(\varphi)=\sum_{\varphi^{h} \geq \varphi} \bigotimes_{p \in P} \bigotimes_{l \in \mathrm{m}} \delta_{p}^{h(l)_{p}}
$$

and, as already noted, write $\bar{R}(\varphi)=\left(n^{\varphi}\right)^{-1} R(\varphi), \varphi \in \operatorname{Hom}(P, P(\mathbf{m}))$. As now defined the space $V$ has a basis consisting of all elements

$$
\bigotimes_{p \in P}\left(\delta_{p}^{i_{p 1}} \otimes \cdots \otimes \delta_{p}^{i_{p m}}\right) \quad\left(i_{p l} \in \mathbf{n}_{p}, l \in \mathbf{m}, p \in P\right) .
$$

For each $p \in P$, we write $\delta_{p}^{\cdot}=n_{p}^{-1}\left(\delta_{p}^{1}+\cdots+\delta_{p}^{n_{p}}\right)$. If $s(p)$ is a subset of $\mathbf{m}$, for each $p \in P$, we define the averaging operator $\theta_{s}$ to be the linear transformation on $V$ which takes the basis elements (3.2) to the elements obtained by replacing $\delta_{p}^{i p l}$ in (3.2) by $\delta_{p}^{\cdot}$ whenever $l \notin s(p)$, and leaving all other factors $\delta_{p}^{i p l}$ unchanged. For example, if $P=\{1,2\}$ with $1<2, m=2, s(1)=\{1\}, s(2)=\{1,2\}$ and we write $i, j, i^{\prime}, j^{\prime}$ instead of $i_{11}, i_{21}$ etc., then $\theta_{s}$ sends $\delta_{1}^{i} \otimes \delta_{1}^{i^{\prime}} \otimes \delta_{2}^{j} \otimes \delta_{2}^{j^{\prime}}$ to $\delta_{1}^{i} \otimes \delta_{1}^{i} \otimes \delta_{2}^{j} \otimes \delta_{2}^{j^{\prime}}$.

DEFINITION 3.1. Let $s \in \operatorname{Hom}(P, B(\mathbf{m}))$. For every $p \in P$ define the element $\gamma_{s} \in \operatorname{Hom}(P, P(\mathbf{m}))$ by taking $\gamma_{s}(p)$ to be the partition of $\mathbf{m}$ whose blocks are the set $s(p)$ (if non-empty), and the singleton set $\{l\}$, for each $l \in \mathbf{m} \backslash s(p)$.

[It is easy to see that $p \rightarrow \gamma_{s}(p)$ is order-preserving since we have assumed that $p \rightarrow s(p)$ is.]

Lemma 3.1. Let $\varphi \in \operatorname{Hom}(P, P(\mathbf{m}))$ and $s \in \operatorname{Hom}(P, B(\mathbf{m}))$ be given. Then

$$
\bar{R}(\varphi)^{\theta_{\bullet}}=\bar{R}\left(\varphi \wedge \gamma_{s}\right)
$$


Proof. We show first that it is enough to prove this for the special case where $|P|=1$.

Let us write $V_{p}=\left(\mathbf{R}^{n_{p}}\right)^{\otimes m}$, for each $p$, so that the space $V$ in which $\bar{R}(\varphi)$ lies in expressible as a tensor product

$$
V=\bigotimes\left\{V_{p}: p \in P\right\}
$$

Each $V_{p}$ is itself the space of tensors associated with $P(\mathbf{m})$ and the single-element indexing poset $\{p\}$. Identifying the components $\varphi(p)$ and $s(p)$ in the natural way with elements of $\operatorname{Hom}(\{p\}, P(\mathbf{m}))$ and $\operatorname{Hom}(\{p\}, B(\mathbf{m}))$, respectively, we may associate with each value of $p$ a tensor $R(\varphi(p))$ and an averaging operator $\theta_{s(p)}$ appropriate to the space $V_{p}$. The reduction to the case $|P|=1$ amounts to showing that the Lemma is true for $\bar{R}(\varphi)$ and $\theta_{s}$ when it is true for $\bar{R}(\varphi(p))$ and $\theta_{s(p)}$, for every $p$.

Now the definition of $R$ shows immediately that

$$
R(\varphi)=\bigotimes\{R(\varphi(p)): p \in P\}
$$

and we obtain the analogous equation for $\bar{R}$ as soon as we observe that the normalizing factor $1 / n^{\varphi}$ for $R(\varphi)$ is the product of the normalizing factors $1 / n_{p}^{\varphi(p)}$ for the $R(\varphi(p))$.

Next recall that the definition of $\theta_{s}$ specified its action on $V$ by stipulating that certain of the terms $\delta_{p}^{i}$ appearing in (3.2) were to be replaced by terms $\delta_{p}^{*}$. For a given $p$, the choice of the terms to be replaced depended only on $s(p)$ and not on the sets $s(q)$ for $q \neq p$. Thus applying $\theta_{s}$ to the element (3.2) is the same as applying $\theta_{s(p)}$ to the factor $\delta_{p}^{i_{p 1}} \otimes \cdots \otimes \delta_{p}^{i_{p m}}$ for each $p$, and consequently

$$
\bar{R}(\varphi)^{\theta_{s}}=\bigotimes\left\{\bar{R}(\varphi(p))^{\theta_{s(p)}}: p \in P\right\} .
$$

Hence we are now reduced to proving that

$$
\bar{R}(\varphi(p))^{\theta_{\theta(p)}}=\bar{R}\left(\left(\varphi \wedge \gamma_{s}\right)(p)\right)=\bar{R}\left(\varphi(p) \wedge \gamma_{s(p)}\right)
$$

for every $p$, which is the required reduction to the case $|P|=1$.

From now on we may accordingly omit any reference to components or the set $P$, and assume that $\varphi \in P(\mathbf{m})$ and $s \subseteq \mathbf{m}$.

First we obtain an expression for the RHS of (3.3). The definition of $\gamma_{s}$ implies that the blocks of $\varphi \wedge \gamma_{s}$ are the blocks of $\varphi \cap s$, together with a singleton block $\{l\}$ for each $l$ in $s^{\prime}=\mathbf{m} \backslash s$. It follows, firstly, that

$$
b\left(\varphi \wedge \gamma_{s}\right)=b(\varphi \cap s)+\left|s^{\prime}\right|
$$

and, secondly, that we can enumerate all possible maps $h: \mathbf{m} \rightarrow \mathbf{n}$ with $\operatorname{ker} h \geq$ $\varphi \wedge \gamma_{s}$ by first enumerating their restrictions $h_{1}: s \rightarrow \mathbf{n}$, which are all possible maps with $\operatorname{ker} h_{1} \geq \varphi \cap s$, and independently enumerating the ways of assigning 
one of the $n$ possible images to each of the singleton blocks $\{l\}$, for $l \in s^{\prime}$. Hence in the expression defining $\bar{R}\left(\varphi \wedge \gamma_{s}\right)$, namely

$$
\left(n^{\varphi \wedge \gamma_{s}}\right)^{-1} \sum_{h} \bigotimes \delta^{h(l)}
$$

where the sum is over all the $h: \mathbf{m} \rightarrow \mathbf{n}$ whose enumeration we have just discussed, we can replace the summation over $h$ by one over all $h_{1}: s \rightarrow \mathrm{n}$ with $\operatorname{ker} h_{1} \geq \varphi \cap s$, together with summations over indices $i_{1}, \ldots, i_{r}$, say, where $r=\left|s^{\prime}\right|$. That is,

$$
\bar{R}\left(\varphi \wedge \gamma_{s}\right)=\left(n^{\varphi \wedge \gamma_{s}}\right)^{-1} \sum_{h_{1}} \sum_{i_{1}} \cdots \sum_{i_{r}} \bigotimes_{l \in s} \delta^{h_{1}(l)} \otimes \delta^{i_{1}} \otimes \cdots \otimes \delta^{i_{r}}
$$

Now (3.4) shows that

$$
n^{\varphi \wedge \gamma_{s}}=n^{\varphi \cap s} n^{r},
$$

so the multiplier outside the summations contains a factor $1 / n$ for each of the summations over $i_{1}, \ldots, i_{r}$. Since

$$
\delta^{\cdot}=n^{-1} \sum_{i} \delta^{i}
$$

we can rewrite the last expression for $\bar{R}\left(\varphi \wedge \gamma_{s}\right)$ to give

$$
\bar{R}\left(\varphi \wedge \gamma_{s}\right)=\left(n^{\varphi \cap s}\right)^{-1} \sum_{h_{1}} \bigotimes_{l \in s} \delta^{h_{1}(l)} \otimes \delta^{*} \otimes \cdots \otimes \delta^{\circ}
$$

where there are $r$ factors $\delta^{\circ}$, and $h_{1}$ satisfies the conditions specified above.

Next we expand the LHS of (3.3). The definitions of $\vec{R}(\varphi)$ and $\theta_{s}$ show that

$$
\bar{R}(\varphi)^{\theta_{0}}=\left(n^{\varphi}\right)^{-1} \sum_{h} \bigotimes_{l \in s} \delta^{h(l)} \otimes \delta^{*} \otimes \cdots \otimes \delta^{*},
$$

where there are again $r$ factors $\delta^{*}$, corresponding to elements of $s^{\prime}$, but the summation is now over all maps $h: \mathbf{m} \rightarrow \mathbf{n}$ with $\operatorname{ker} h \geq \varphi$. Since the value of each summand in (3.6) depends only on the values of $h$ on $s$, the summands in (3.6) are the same as those in (3.5), but each one in (3.5) will appear in (3.6) as many times as there are different maps $h: \mathbf{m} \rightarrow \mathbf{n}$ whose restriction to $s$ agrees with a given $h_{1}$. Now given any such $h$, we obtain all other maps with the same restriction by redefining $h$ on those blocks of $\varphi$ that do not intersect with $s$, in all possible ways. The number of such blocks is $b(\varphi)-b(\varphi \cap s)$, so each summand in (3.5) appears $n^{\varphi} / n^{\varphi n s}$ times in (3.6). But this last number is precisely the ratio of the multipliers in (3.5) and (3.6), so these expressions have the same value and the proof is complete.

This lemma shows the importance of the elements $\gamma_{s}$, and in particular of the elements $\varphi \wedge \gamma_{s}$ associated with a given $\varphi \in \operatorname{Hom}(P, P(\mathbf{m}))$ and $s \in$ $\operatorname{Hom}(P, B(\mathbf{m}))$. Note that we can have $\varphi \wedge \gamma_{s_{1}}=\varphi \wedge \gamma_{s_{2}}$ with $s_{1} \neq s_{2}$. We 
define a new partial order $\leq^{*}$ on $\operatorname{Hom}(P, P(\mathbf{m}))$ by writing $\psi \leq^{*} \varphi$ if and only if $\psi=\varphi \wedge \gamma_{s}$ for some $s \in \operatorname{Hom}(P, B(\mathbf{m}))$. Letting $\mu *$ and $\zeta^{*}$ denote the Möbius and zeta functions of $\left(\operatorname{Hom}(P, P(m)), \leq^{*}\right)$ (see the Appendix), we can now define the main objects of interest in this section.

$$
T(\varphi)=\sum_{\psi} \mu^{*}(\psi, \varphi) \bar{R}(\psi)
$$

By Möbius inversion we immediately have

$$
\bar{R}(\varphi)=\sum_{\psi} \varsigma^{*}(\psi, \varphi) T(\psi)
$$

Here it is implicit that the sums are over all $\psi \in \operatorname{Hom}(P, \mathcal{P}(\mathbf{m}))$.

We now turn to alternative formulae for the tensors $T(\varphi)$, the last one being the most recognizable and most useful one.

For $\varphi \in \operatorname{Hom}(P, \mathcal{P}(\mathbf{m}))$ define $L_{\varphi} \in \operatorname{Hom}(P, B(\mathbf{m}))$ by $l \in L_{\varphi}(p)$ if and only if $\{l\}$ is not a block of $\varphi(p)$, that is, $L_{\varphi}(p)$ is the union of the non-singleton blocks of $\varphi(p)$. Also let $S_{\varphi}$ be the subset of $\operatorname{Hom}(P, B(\mathbf{m}))$ of all elements $s$ which satisfy $\bigcup\left\{L_{\varphi}(q): q<p\right\} \subseteq s(p) \subseteq L_{\varphi}(p)$ for all $p \in P$. In the lemma below $\tilde{\mu}$ denotes the Möbius function of the poset $\operatorname{Hom}(P, B(\mathbf{m}))$.

LEMMA 3.2. The following are alternative expressions for $T(\varphi)$ :

(i) $T(\varphi)=\sum_{s} \tilde{\mu}\left(s, L_{\varphi}\right) \bar{R}\left(\varphi \wedge \gamma_{s}\right)$

where the sum is over all $s \in \operatorname{Hom}(P, B(\mathbf{m}))$.

(ii) $T(\varphi)=\sum_{s \in S_{\varphi}}(-1)^{d_{\varphi}(s)} \bar{R}\left(\varphi \wedge \gamma_{s}\right)$ where $d_{\varphi}(s)=\sum_{p}\left|L_{\varphi}(p) \backslash s(p)\right|$.

PrOOF. By the definition of $T(\varphi)$, it is enough to show that

$$
\mu^{*}(\psi, \varphi)=\sum_{s} \tilde{\mu}\left(s, L_{\varphi}\right)
$$

$\sum_{s}$ denoting the sum over all $s$ with $\varphi \wedge \gamma_{s}=\psi$. To do this, we form the product of the RHS with $\varsigma^{*}(\chi, \psi)$, sum over all $\psi \in \operatorname{Hom}(P, \mathcal{P}(\mathbf{m}))$, and find that the result is $\delta(\chi, \varphi)=1$ if $\chi=\varphi$, and zero otherwise. Indeed we have

$$
\sum_{\psi} \varsigma^{*}(\chi, \psi) \sum_{s} \tilde{\mu}\left(s, L_{\varphi}\right)=\sum\left\{\tilde{\mu}\left(s, L_{\varphi}\right): \varphi \wedge \gamma_{s} \geq \chi\right\},
$$

where the last sum is over $s$ with $\varphi \wedge \gamma_{s}=\psi$. Now it follows immediately from the definitions that for any $s, \chi$ we have $\gamma_{s} \geq \chi$ iff $s \geq L_{\chi}$. The LHS of the last equation is clearly zero unless $\chi \leq \varphi$, so the condition $\varphi \wedge \gamma_{s} \geq \chi$ may be replaced by $s \geq L_{\chi}$. Also we have $\tilde{\mu}\left(s, L_{\varphi}\right)=0$ whenever $s \nless L_{\varphi}$, so we can rewrite the above sum as

$$
\sum_{L_{\chi} \leq s \leq L_{\varphi}} \tilde{\mu}\left(s, L_{\varphi}\right)
$$


which is equal to $\delta(\chi, \varphi)$ by elementary properties of the Möbius function. This proves (i).

Turning now to the proof of (ii), we observe that it is enough to show that

$$
\tilde{\mu}\left(s, L_{\varphi}\right)= \begin{cases}(-1)^{d_{\varphi}(s)} & \text { if } s \in S_{\varphi} \\ 0 & \text { otherwise }\end{cases}
$$

Using the formula in Speed (1984) for the Möbius function of a Hom poset, we have

$$
\tilde{\mu}\left(s, L_{\varphi}\right)=\prod_{p \in P}\left\{\mu_{B(m)}\left(s(p), L_{\varphi}(p)\right) \prod_{q>p} \varsigma_{B(\mathbf{m})}\left(L_{\varphi}(p), s(q)\right)\right\}
$$

and the second product is zero unless $s(q) \geq L_{\varphi}(p)$ whenever $q<p$, that is, unless $s(p) \supseteq \bigcup\left\{L_{\varphi}(q): q<p\right\}$ for every $p$ in $P$. Furthermore, by the wellknown formula for the Möbius function of $B(\mathbf{m})$, we have

$$
\mu_{B(\mathbf{m})}\left(s(p), L_{\varphi}(p)\right)= \begin{cases}0 & \text { if } s(p) \nsubseteq L_{\varphi}(p), \\ (-1)^{\left|L_{\varphi}(p) \backslash s(p)\right|} & \text { otherwise. }\end{cases}
$$

From this the result follows immediately.

Our final expressions for $T(\varphi)$ include one which can be related to the decomposition of a $G W(\mathbf{I})$-invariant array into its orthogonal projections onto the irreducible invariant subspaces of the natural permutation representation of the group $G W(\mathbf{I})$. We give the details in the next section.

For $\varphi \in \operatorname{Hom}(P, \mathcal{P}(\mathbf{m}))$ and $l \in \mathbf{m}$ write $a_{\varphi}(l)$ for the set of $p \in P$ for which $\{l\}$ is not a block of $\varphi(p)$. Recalling the definition of $L_{\varphi}(p)$ given just before the last lemma, we note that $p \in a_{\varphi}(l)$ if and only if $l \in L_{\varphi}(p)$. As a further piece of notation write $a_{\varphi}^{*}(l)$ for the minimal elements of $a_{\varphi}(l)$ (as a sub poset of $P$ ) and $L_{\varphi}^{*}(p)=L_{\varphi}(p) \backslash \bigcup\left\{L_{\varphi}(q): q<p\right\}$.

LEMMA 3.3. (i) In the notation of the present section, $n^{\varphi} T(\varphi)$ may be written as the sum over all $h: \mathbf{m} \rightarrow \prod\left\{\mathbf{n}_{p}: p \in P\right\}$ such that $\varphi^{h} \geq \varphi$ of the terms

$$
\bigotimes_{p \in P}\left\{\bigotimes_{l \in L_{\varphi}(p) \backslash L_{\dot{\varphi}}^{*}(p)} \delta_{p}^{h(l) p} \otimes \bigotimes_{l \in L_{\varphi}^{*}(p)}\left(\delta_{p}^{h(l)_{p}}-\delta_{p}^{*}\right) \otimes \bigotimes_{l \notin L_{\varphi}(p)} \delta_{p}^{\cdot}\right\} .
$$

(ii) Moreover, if we rearrange the order of tensoring to that used in III, cf. the remark at the beginning of this section, then $n^{\varphi} T(\varphi)$ can be written as the sum over all $h$ with $\varphi^{h} \geq \varphi$ of the terms

$$
\bigotimes_{l \in \mathbf{m}}\left\{\bigotimes_{p \in a_{\varphi}(l) \backslash a_{\varphi}^{*}(l)} \delta_{p}^{h(l)_{p}} \otimes \bigotimes_{p \in a_{\varphi}^{*}(l)}\left(\delta_{p}^{h(l)_{p}}-\delta_{p}^{*}\right) \otimes \bigotimes_{p \notin a_{\varphi}(l)} \delta_{p}^{\cdot}\right\} .
$$

PROOF. The equivalent of the two formulae is immediate, for we have already observed that $p \in a_{\varphi}(l)$ if and only if $l \in L_{\varphi}(p)$, and it is easy to see that 
$p \in a_{\varphi}^{*}(l)$ if and only if $l \in L_{\varphi}^{*}(p)$. To derive (i) we expand the expression in braces, obtaining

$$
\bigotimes_{p \in P}\left\{\sum_{s(p)}(-1)^{\left|L_{\varphi}(p) \backslash s(p)\right|}\left[\bigotimes_{l \in s(p)} \delta_{p}^{h(l)_{p}} \otimes \bigotimes_{l \notin s(p)} \delta_{p}^{\cdot}\right]\right.
$$

where $s(p)$ ranges over all subsets of $\mathbf{m}$ with $\bigcup\left\{L_{\varphi}(q): q<p\right\} \subseteq s(p) \subseteq L_{\varphi}(p)$. But this last condition is precisely that occurring in the definition of $S_{\varphi}$, so that, if we multiply the sum under discussion by $\left(n^{\varphi}\right)^{-1}$, we can write the resulting expression as

$$
\begin{aligned}
\left(n^{\varphi}\right)^{-1} & \sum_{\varphi^{h} \geq \varphi} \sum_{s \in S_{\varphi}} \bigotimes_{p \in P}(-1)^{\left|L_{\varphi}(p) \backslash s(p)\right|}\left[\bigotimes_{l \in s(p)} \delta_{p}^{h(l)_{p}} \otimes \bigotimes_{l \notin s(p)} \delta_{p}^{\cdot}\right] \\
& =\sum_{s \in S_{\varphi}}(-1)^{d_{\varphi}(s)}\left(n^{\varphi}\right)^{-1} \sum_{\varphi^{h} \geq \varphi} \bigotimes_{p \in P}\left[\bigotimes_{l \in s(p)} \delta_{p}^{h(l)_{p}} \otimes \bigotimes_{l \notin s(p)} \delta_{p}^{\cdot}\right] \\
& =\sum_{s \in S_{\varphi}}(-1)^{d_{\varphi}(s)} \bar{R}(\varphi)^{\theta_{\varphi}} \quad \text { by definition } \\
& =\sum_{s \in S_{\varphi}}(-1)^{d_{\varphi}(s)} \bar{R}\left(\varphi \wedge \gamma_{s}\right) \quad \text { by Lemma 3.1 } \\
= & T(\varphi) \quad \text { by Lemma 3.2. }
\end{aligned}
$$

\section{Statistical interpretation of the $T(\varphi)$}

The tensors $T(\varphi)$ have an important statistical interpretation which is not immediately evident from what we have given so far. In order to obtain this interpretation and to see why the associated symmetric functions $t(\varphi)=[T(\varphi) \mid X]$ will be readily computed in a statistical context we reexamine the previous discussion in the special case $m=2$ and relate this to the material in III, Section 6.

Let us begin by noting that when $m=2$ the partial orders $\leq$ and $\leq^{*}$ coincide. This is an immediate consequence of the fact that every element $\varphi \in \operatorname{Hom}(P, P(2))$ has the form $\gamma_{s}$ for a suitable $s \in \operatorname{Hom}(P, B(2))$. Indeed $s=L_{\varphi}$ will do, for it is easily checked from the definitions that $L_{\varphi}(p)=\{1,2\}$ if and only if $\varphi(p)=12$ (the larger element of $P(2)$ ). Further, it can be seen that when $m=2$ the sets $a_{\varphi}(1)$ and $a_{\varphi}(2)$ must coincide, equalling $a_{\varphi}=\{p \in$ $P: \varphi(p)=12\}$, say. In fact, the mapping $\varphi \rightarrow a_{\varphi}$ is an isomorphism between $\operatorname{Hom}(P, P(2))$ and the poset $F(P)$ of all filters on $P$ (that is, subsets $a \subseteq P$ with the property that $p \in a$ and $q>p$ implies $q \in a$ ). It follows from these remarks 
that any discussion of the tensors $T(\varphi)$ when $m=2$ can be expressed equally well in terms of elements $\varphi \in \operatorname{Hom}(P, P(2))$ or filters $a \in F(P)$.

In III, Section 6 we took the opportunity of regarding our tensors as $n \times n$ matrices when $m=2$ (where $n=|\mathbf{I}|$ ) and it is convenient to do so here as well. Formula (6.1) of III defined certain symmetric idempotent matrices $\left\{S_{\sigma}\right\}$ in terms of the underlying associated matrices $\left\{A_{\rho}\right\}$ and certain structural constants $\left(q_{\rho \sigma}\right)$. The $S_{\sigma}$ are called the stratum projectors and they play a key role in the analysis of variance, see Speed $(1985,1986 \mathrm{c})$ and Bailey et al. (forthcoming), and they will turn out to be the same as the $T$-tensors when $m=2$. Furthermore, we will see that in a sense the general $T$-tensors are polynomials intimately related to those of order 2 . For the next result we suppose that $m=2$ and write our tensors as matrices. In this case the expressions equivalent to those derived in the previous section may be rederived using different methods.

Proposition 4.1. For all $\sigma \in \operatorname{Hom}(P, P(2))$ we have

(i) $S_{\sigma}=n \sum_{\pi} \mu(\pi, \sigma) \bar{R}_{\pi}$.

In particular, $T(\sigma)=n^{-1} S_{\sigma}$.

(ii) $S_{\sigma}=n \sum_{\pi}(-1)^{d(\pi, \sigma)} \bar{R}_{\pi}$

where the sum is only over those $\pi$ for which $D(\pi, \sigma)=\{p \in P: \pi(p)=$ $1 \mid 2, \sigma(p)=12\}$ has only pairwise incomparable elements, and $d(\pi, \sigma)=|D(\pi, \sigma)|$.

(iii) With its rows and columns suitably ordered, the following is an expression for $S_{\sigma}$ :

$$
\bigotimes_{p \in a(\sigma) \backslash a^{*}(\sigma)} I_{n_{p}} \otimes \bigotimes_{p \in a^{*}(\sigma)}\left(I_{n_{p}}-n_{p}^{-1} J_{n_{p}}\right) \otimes \bigotimes_{p \notin a(\sigma)} n_{p}^{-1} J_{n_{p}}
$$

where $a(\sigma)=\{p \in P: \sigma(p)=12\}, a^{*}(\sigma)$ is the set of minimal elements of $a(\sigma)$, $I_{n_{p}}$ and $J_{n_{p}}$ are the $n_{p} \times n_{p}$ identity matrix and matrix of all $1 s$, respectively.

ProOF. (i) If we take equation (6.1) of III defining $S_{\sigma}$, use the representation of $A_{\rho}$ in terms of the relationship matrices $\left\{R_{\tau}\right\}$, and the equation from Speed and Bailey (1982) expressing $\left(q_{\rho \sigma}\right)$ in terms similar to that of $\operatorname{III}(6.4)$, we find

$$
\begin{aligned}
S_{\sigma} & =n^{-1} \sum_{\rho} q_{\rho \sigma} A_{\rho} \\
& =n^{-1} \sum_{\rho}\left\{\sum_{\pi} \mu(\pi, \sigma) \zeta(\pi, \rho) n_{\pi}\right\} \sum_{\tau} \mu(\rho, \tau) R_{\tau} \\
& =n^{-1} \sum_{\pi} \mu(\pi, \sigma) n_{\pi} R_{\pi}
\end{aligned}
$$

summing out $\rho$ and forcing $\tau=\pi$. But $n^{-1} n_{\pi} R_{\pi}=n \bar{R}_{\pi}$ as defined earlier, and so (i) is proved. Note that the RHS is just the definition of $T(\sigma)$ given in the previous section, for we have already noted that $\leq$ and $\leq^{*}$ coincide when $m=2$. 
(ii) This expression follows readily from (i) using the formula given in Speed (1984) for the Möbius function $\mu$ of $\operatorname{Hom}(P, P(2))$. It is the version of Lemma 3.2(ii) appropriate in this case. Lemma 3.2(i) is really nothing more than (i) above partially expressed in terms of the filters, and we remark that both (i) and (ii) could be re-expressed wholly in terms of the lattice $F(P)$ of all filters of $P$.

(iii) This formula is the analogue of Lemma 3.3(ii) and it is proved in a manner identical to that of Lemma 3.3(i). We omit the details.

With this proposition proved we can begin to make the connection between the symmetric functions defined by the tensors $T(\varphi)$ and those of order 2 . The key result is embodied in the following Corollary to Proposition 4.1 where we use notation for the symmetric functions introduced in II, Section 2 and III, Section 2.

COROllaRY. For any array $x=\left(x_{i}: i \in \mathbf{I}\right)$ of indeterminates, $\sigma \in$ $\operatorname{Hom}(P, P(\mathbf{2}))$ and element $i=\left(i_{p}: p \in P\right) \in \mathbf{I}=\prod\left\{\mathbf{n}_{p}: p \in P\right\}$ we have

$$
\left(S_{\sigma} x\right)_{i}=\left[\bigotimes_{p \in a(\sigma) \backslash a^{*}(\sigma)} \delta_{p}^{i_{p}} \otimes \bigotimes_{p \in a^{*}(\sigma)}\left(\delta_{p}^{i_{p}}-\delta_{p}^{*}\right) \otimes \bigotimes_{p \notin a(\sigma)} \delta_{p}^{*} \mid x\right]
$$

PROOF. This is immediate from expression (iii) of the proposition. Comparing this expression with Lemma 3.3(ii) gives us the result we have been working towards.

THEOREM 4.1. For any array $x=\left(x_{i}(l): l \in \mathbf{m}, i \in \mathrm{I}\right)$ viewed as an Iindexed array of $m$-tuples of indeterminates or as $m$ arrays of indeterminates indexed by $I$, and for all $\varphi \in \operatorname{Hom}(P, P(\mathbf{m}))$ we have

$$
[T(\varphi) \mid x]=\left(n^{\varphi}\right)^{-1} \sum_{h: \varphi^{h} \geq \varphi} \prod_{l \in \mathbf{m}}\left(S_{a_{\varphi}(l)} x(l)\right)_{h(l)} .
$$

(Here we use the isomorphism between $\operatorname{Hom}(P, P(2))$ and $F(P)$ mentioned above to label $S_{a_{\varphi}(l)}$ by the filter $a_{\varphi}(l)$, rather than by the element of $\operatorname{Hom}(P, P(2))$ to which it corresponds.)

Thus the symmetric functions defined by the tensors $T(\varphi)$ are essentially polynomials of degree $m$ in the expressions in the stratum projections $S_{a} x(l)$, $a \in F(P), l \in \mathbf{m}$, of the arrays $x(l)$.

EXAMPLES. Expressions for the $T(\varphi)$ are most readily given via equation (4.1). One uses the definition of $a_{\varphi}(l)$ for each $l \in \mathbf{m}$ and assigns equality or inequality of multi-indices $\{h(l): l \in \mathbf{m}\}$ on the basis of $\varphi$. Note that the $t$ 's are properly normalized. 
(a) We begin with some examples in which $P$ has only a single element, 1 say. Putting $i_{1}=i, n_{1}=n$ and writing $W_{i}, X_{i}, Y_{i}$ and $Z_{i}$ instead of $X_{i}(1), X_{i}(2)$, $X_{i}(3)$ and $X_{i}(4)$ we obtain the following expressions.

For order $m=2$ we have

$$
\begin{aligned}
t(12) & =n^{-1} \sum_{i}\left(W_{i}-W \cdot\right)\left(X_{i}-X .\right) \\
t(1 \mid 2) & =W \cdot X .
\end{aligned}
$$

For order $m=3$ we have

$$
\begin{aligned}
t(123) & =n^{-1} \sum_{i}\left(W_{i}-W .\right)\left(X_{i}-X .\right)\left(Y_{i}-Y .\right), \\
t(12 \mid 3) & =n^{-1} \sum_{i}\left(W_{i}-W .\right)\left(X_{i}-X .\right) Y . \text { and two similar, and } \\
t(1|2| 3) & =W \cdot X . Y .
\end{aligned}
$$

Finally, for order $m=4$ we have

$$
\begin{aligned}
t(1234) & =n^{-1} \sum_{i}\left(W_{i}-W .\right)\left(X_{i}-X .\right)\left(Y_{i}-Y .\right)\left(Z_{i}-Z .\right) \\
t(123 \mid 4) & =n^{-1} \sum_{i}\left(W_{i}-W .\right)\left(X_{i}-X .\right)\left(Y_{i}-Y .\right) Z . \text { and three similar, } \\
t(12 \mid 34) & =n^{-2} \sum_{i} \sum_{i^{\prime}}\left(W_{i}-W .\right)\left(X_{i}-X .\right)\left(Y_{i^{\prime}}-Y .\right)\left(Z_{i^{\prime}}-Z .\right) \text { and two similar, } \\
t(12|3| 4) & =n^{-1} \sum_{i}\left(W_{i}-W_{.}\right)\left(X_{i}-X .\right) Y . Z . \text { and five similar, and } \\
t(1|2| 3 \mid 4) & =W . X . Y . Z .
\end{aligned}
$$

(b) Turning now to the case where $P$ has two comparable elements 1 nesting 2 , we use $i$ and $j$ instead of $i_{1}$ and $i_{2}$, and $m=n_{1}, n=n_{2}$.

$m=2$.

$$
\begin{aligned}
t(12,12) & =m^{-1} n^{-1} \sum_{i} \sum_{j}\left(W_{i j}-W_{i}\right)\left(X_{i j}-X_{i} \cdot\right) \\
t(12,1 \mid 2) & =m^{-1} \sum_{i}\left(W_{i}-W_{. .}\right)\left(X_{i} \cdot-X . .\right) \\
t(1|2,1| 2) & =W . . X . .
\end{aligned}
$$


$m=3$ : selected examples.

$$
\begin{aligned}
& t(123,123)=m^{-1} n^{-1} \sum_{i} \sum_{j}\left(W_{i j}-W_{i} \cdot\right)\left(X_{i j}-X_{i} \cdot\right)\left(Y_{i j}-Y_{i} \cdot\right), \\
& t(123,12 \mid 3)=m^{-1} n^{-1} \sum_{i} \sum_{j}\left(W_{i j}-W_{i} \cdot\right)\left(X_{i j}-X_{i} \cdot\right)\left(Y_{i}-Y_{. .}\right), \\
& \left.t(123,1|2| 3)=m^{-1} \sum_{i}\left(W_{i}-W_{. .}\right)\left(X_{i} \cdot-X . .\right) Y_{i}-Y_{. .}\right), \\
& t(12|3,12| 3)=m^{-1} n^{-1} \sum_{i} \sum_{j}\left(W_{i j}-W_{i} \cdot\right)\left(X_{i j}-X_{i} \cdot\right) . \cdot, \\
& t(12|3,1| 2 \mid 3)=m^{-1} \sum_{i}\left(W_{i \cdot}-W . .\right)\left(X_{i \cdot}-X . .\right) Y . ., \\
& t(1|2| 3,1|2| 3)=W . . X . . Y . .
\end{aligned}
$$

$m=4$ : selected examples.

$$
\begin{aligned}
& t(1234,1234)=m^{-1} n^{-1} \sum_{i} \sum_{j}\left(W_{i j}-W_{i} \cdot\right)\left(X_{i j}-X_{i}\right)\left(Y_{i j}-Y_{i \cdot}\right)\left(Z_{i j}-Z_{i} \cdot\right), \\
& t(1234,12 \mid 34)=m^{-1} n^{-2} \sum_{i} \sum_{j} \sum_{j^{\prime}}\left(W_{i j}-W_{i} \cdot\right)\left(X_{i j}-X_{i} \cdot\right) \\
& \times\left(Y_{i j^{\prime}}-Y_{i} \cdot\right)\left(Z_{i j^{\prime}}-Z_{i} \cdot\right), \\
& t(1234,12|3| 4)=m^{-1} n^{-1} \sum_{i} \sum_{j}\left(W_{i j}-W_{i}\right)\left(X_{i j}-X_{i \bullet}\right)\left(Y_{i \bullet}-Y_{. .}\right)\left(Z_{i}-Z . .\right), \\
& t(1234,1|2| 3 \mid 4)=m^{-1} \sum_{i}\left(W_{i \cdot}-W_{. .}\right)\left(X_{i \cdot}-X . .\right)\left(Y_{i}-Y_{. .}\right)\left(Z_{i \bullet}-Z_{. .}\right), \\
& t(123|4,12| 3 \mid 4)=m^{-1} n^{-1} \sum_{i} \sum_{j}\left(W_{i j}-W_{i} \cdot\right)\left(X_{i j}-X_{i} \cdot\right)\left(Y_{i} \cdot-Y_{. .}\right) Z \ldots
\end{aligned}
$$

(c) Finally, we consider briefly the case where $P$ has two incomparable elements 1 and 2 and again we use $i, j, m$ and $n$ instead of $i_{1}, i_{2}, n_{1}$ and $n_{2}$. $m=3$ : selected examples.

$$
\begin{array}{r}
t(123,123)=m^{-1} n^{-1} \sum_{i} \sum_{j}\left(W_{i j}-W_{i \cdot}-W_{\cdot j}+W_{. .}\right)\left(X_{i j}-X_{i \cdot}-X_{\cdot j}+X . .\right) \\
\times\left(Y_{i j}-Y_{i \cdot}-Y_{\cdot j}+Y_{. .}\right), \\
t(12|3,13| 2)=m^{-1} n^{-1} \sum_{i} \sum_{j}\left(W_{i j}-W_{i \cdot}-W_{\cdot j}+W_{. \cdot}\right)\left(X_{i \cdot}-X . .\right)\left(Y_{\cdot j}-Y . .\right) .
\end{array}
$$

$m=4:$ an example.

$$
\begin{aligned}
t(12|3| 4,13|2| 4)=m^{-1} n^{-1} \sum_{i} \sum_{j}\left(W_{i j}-W_{i \cdot}-\right. & \left.W_{\cdot j}+W_{. .}\right) \\
& \times\left(X_{i \cdot}-X . .\right)(Y \cdot j-Y . .) Z . .
\end{aligned}
$$


REMARK. Although it is the generalized $k$-statistics $F(\varphi)$ and their associated functions $k(\varphi)=[F(\varphi) \mid X]$ and $f(\varphi)=\mathrm{E}\{k(\varphi)\}$ which have the more fundamental statistical importance, it is quite clear that the expressions $t(\varphi)$ are statistically meaningful. For example, it is obvious that $t(123,123)$ and $t(1234,1234)$ in (b) above relate to within class (cross) skewness or, following Tukey, skewmulance, whilst $t(1234,1234)$ is clearly connected with within class kurtosis. Similarly $t(123,1|2| 3)$ and $t(1234,1|2| 3 \mid 4)$ (not given) would relate to between class skewness and kurtosis, respectively. Perhaps more interestingly, $t(12|3,13| 2)$ in (c) above-whose resemblance to Tukey's one degree of freedom for non-additivity can hardly be missed-is closely connected with nonadditivity. It is a key component in $k(12|3,13| 2)$ which estimates a non-additivity measure $f(12|3,13| 2)$ in two-way structures. The details of this connection will be explained elsewhere, although that use of $k$ 's, $f$ 's and $t$ 's to measure nonadditivity (and inhomogeneity) will be discussed further in paper VI in this series.

\section{Formulae for generalized $k$-statistics: theory}

Having introduced the tensors $T(\varphi)$ it now remains to give the formulae expressing the generalized $k$-statistics $F(\varphi)$ in terms of them. Our main result is trivial to prove; what is incredible is the way the double sum collapses to a modest number of terms in all cases of interest.

PROPOSITION 5.1. The transition matrix $M(F, T)$ expressing generalized $k$ statistics $F$ in terms of tensors $T(\varphi)$ has entries

$$
M(F, T)_{\varphi, \psi}=\sum_{\tau} \frac{\mu(\tau, \varphi)}{(n)_{\tau}}\left(\sum_{\chi} \mu(\tau, \chi) \zeta^{*}(\chi, \psi) n^{\chi}\right) .
$$

ProOF. This is an immediate consequence of Proposition 2.1 of III, which gives $M(F, R)$ (actually found in II) and the formula for $M(R, T)$ derived in Section 3 above.

In general (5.1) does not simplify directly unless $m=2$; there the explicit formula for $\mu$ and the fact that $\varsigma^{*}=\varsigma$ combine to give the expression of Proposition 6.1 of III. In all other cases the evaluation of (5.1) seems to be a lengthy task and even for the simplest posets $P$ we have had to resort to a computer. The expressions (5.1) seem to have the unusual property of simplifying incredibly, given how complicated they could be, but not sufficiently so that one feels simple formulae exist which could be derived without the use of a computer. This will become clear when we give some of our results in the next section.

Before turning to the examples, we present one further useful result. 
PROPOSITION 5.2. If $M$ denotes the transition matrix $M(F, T)$ corresponding to the poset $P$ consisting of a single element, then the transition matrix $M(F, T)$ corresponding to the poset consisting of two (or more, pairwise) incomparable elements is the tensor product of two (or more) copies of $M$.

PrOOF. This follows directly from (5.1) as soon as we realize that for a poset of two (or more, pairwise) incomparable elements, all of the expressions $n^{\psi},(n)_{\tau}$, $\mu$ and $\varsigma^{*}$ and the elements $\tau, \chi$ of the Hom sets, factor into products of the terms corresponding to the single element poset.

\section{Formulae for generalized $k$-statistics: examples with $m \leq 4$}

In what follows "term $+k$ sim." denotes the sum of all $k+1$ terms based upon similar partitions. We resume the abbreviation $(\varphi)$ for tensor $F(\varphi)$ adopted in III and similarly abbreviate $T(\varphi)$ by $\{\varphi\}$. Brackets to play the role of separators are denoted [ ]. All of the expressions given will be used or referred to in the next paper in this series.

6.1. A single index. We begin with the poset $P$ consisting of a single element 1 with $n=n_{1}$.

$m=2$.

i.

$$
(12)=\frac{n^{2}}{(n)_{2}}\{12\}
$$

ii.

$$
(1 \mid 2)=-\frac{n}{(n)_{2}}\{12\}+\{1 \mid 2\}
$$

$m=3$.

i.

$$
(123)=\frac{n^{3}}{(n)_{3}}\{123\}
$$

ii.

$$
(12 \mid 3)=\frac{n^{2}}{(n)_{2}}\{12 \mid 3\}-\frac{n^{2}}{(n)_{3}}\{123\}
$$

iii. $\quad(1|2| 3)=\{1|2| 3\}-\frac{n}{(n)_{2}}[\{1|2| 3\}+2$ sim. $]+\frac{2 n}{(n)_{3}}\{123\}$.

$m=4$.

i. $\quad(1234)=\frac{n^{3}(n+1)}{(n)_{4}}\{1234\}-\frac{n^{3}(n-1)}{(n)_{4}}[\{12 \mid 34\}+2$ sim. $]$. 
ii. $\quad(123 \mid 4)=-\frac{n^{2}(n+1)}{(n)_{4}}\{1234\}+\frac{n^{2}(n-1)}{(n)_{4}}[\{12 \mid 34\}+2$ sim. $]$

$$
+\frac{n^{3}}{(n)_{3}}\{123 \mid 4\} \text {. }
$$

iii.

$$
\begin{aligned}
(12 \mid 34)= & \frac{n^{2}(n-1)}{(n)_{4}}\{1234\}+\frac{n^{2}}{(n)_{4}}[\{12 \mid 34\}+2 \text { sim. }] \\
& +\frac{n^{3}}{(n)_{3}}\{12 \mid 34\} .
\end{aligned}
$$

iv.

$$
\begin{aligned}
(12|3| 4)= & \frac{2 n^{2}}{(n)_{4}}\{1234\}-\frac{n^{2}}{(n)_{4}}[\{12 \mid 34\}+2 \text { sim. }] \\
& +\frac{n^{2}}{(n)_{3}}\{12 \mid 34\}-\frac{n^{2}}{(n)_{3}}[\{123 \mid 4\}+\{124 \mid 3\}] \\
& +\frac{n^{2}}{(n)_{2}}\{12|3| 4\} .
\end{aligned}
$$

$$
\text { v. } \quad \begin{aligned}
(1|2| 3 \mid 4)= & -\frac{6 n}{(n)_{4}}\{1234\}+\frac{n^{2}}{(n)_{4}}[\{12 \mid 34\}+2 \operatorname{sim} .] \\
& +\frac{2 n}{(n)_{3}}[\{123 \mid 4\}+3 \operatorname{sim} .] \\
& -\frac{n}{(n)_{2}}[\{12|3| 4\}+5 \operatorname{sim} .]+\{1|2| 3 \mid 4\} .
\end{aligned}
$$

6.2. Two crossed indices. We can obtain the corresponding results for the simple crossed structure, that is, $P$ consisting of two incomparable elements, by making use of the rule of Proposition 5.2. For example, if $n_{1}=m$ and $n_{2}=n$, we can use the results of (6.1) above to calculate

$$
\begin{aligned}
(12|3,13| 2)= & \frac{m^{2} n^{2}}{(m)_{2}(n)_{2}}\{12|3,13| 2\}-\frac{m^{2} n^{2}}{(m)_{3}(n)_{2}}\{123,13 \mid 2\} \\
& -\frac{m^{2} n^{2}}{(m)_{2}(n)_{3}}\{12 \mid 3,123\}+\frac{m^{2} n^{2}}{(m)_{3}(n)_{3}}\{123,123\} .
\end{aligned}
$$

6.3. Two nested indices. Our next example gives results for the case where $P$ is the two-element chain corresponding to two indices, one nested within the other. Let $n_{1}=m$ and $n_{2}=n$.

$m=2$.

i.

$$
(12,12)=\frac{n^{2}}{(n)_{2}}\{12,12\}
$$

ii.

$$
(12,1 \mid 2)=-\frac{n}{(n)_{2}}\{12,12\}+\frac{m^{2} n^{2}}{(m)_{2}}\{12,1 \mid 2\} \text {. }
$$


$m=3$. Note that $|\operatorname{Hom}(P, P(3))|=12$ when $P$ is the two-element chain.

i.

$$
(123,123)=\frac{n^{3}}{(n)_{3}}\{123,123\} \text {. }
$$

ii.

$$
(123,12 \mid 3)=-\frac{n^{2}}{(n)_{3}}\{123,123\}+\frac{m^{2} n^{2}}{(m)_{2}(n)_{2}}\{123,12 \mid 3\} .
$$

iii. $\quad(123,1|2| 3)=\frac{2 n}{(n)_{3}}\{123,123\}-\frac{m^{2} n}{(m)_{2}(n)_{2}}[\{123,12 \mid 3\}+2 \operatorname{sim}$. $]$

$$
+\frac{m^{3}}{(m)_{3}}\{123,1|2| 3\} \text {. }
$$

iv. $\quad(12|3,12| 3)=-\frac{m n^{2}}{(m)_{2}(n)_{2}}\{123,12 \mid 3\}+\frac{n^{2}}{(n)_{2}}\{12|3,12| 3\}$.

v. $\quad(12|3,1| 2 \mid 3)=\frac{m n}{(m)_{2}(n)_{2}}\{123,12 \mid 3\}-\frac{m^{2}}{(m)_{3}}\{123,1|2| 3\}$

$$
-\frac{m^{2}}{(m)_{2}}\{12|3,12| 3\}+\frac{m^{2}}{(m)_{2}}\{12|3,1| 2 \mid 3\} \text {. }
$$

vi. $\quad(1|2| 3,1|2| 3)=\frac{2 m}{(m)_{3}}\{123,1|2| 3\}-\frac{m}{(m)_{2}}[\{12|3,1| 2 \mid 3\}+2$ sim. $]$ $+\{1|2| 3,1|2| 3\}$.

$m=4$. Note that $|\operatorname{Hom}(P, P(4))|=60$ when $P$ is the two-element chain.

i. $(1234,1234)=\frac{n^{3}(n+1)}{(n)_{4}}\{1234,1234\}-\frac{n^{3}(n-1)}{(n)_{4}}[\{1234,12 \mid 34\}+2$ sim. $]$.

ii.

$$
\begin{aligned}
(1234,123 \mid 4)= & -\frac{n^{2}(n+1)}{(n)_{4}}\{1234,1234\} \\
& +\frac{n^{2}(n-1)}{(n)_{4}}[\{1234,12 \mid 34\}+2 \text { sim. }] \\
& +\frac{m^{2} n^{3}}{(m)_{2}(n)_{3}}\{1234,123 \mid 4\}
\end{aligned}
$$

iii. $(1234,12 \mid 34)=-\frac{n^{2}(n-1)}{(n)_{4}}\{1234,1234\}$

$$
\begin{aligned}
& +\left[\frac{m n^{4}}{(m)_{2}(n)_{2}^{2}}+\frac{n^{3}}{(n)_{3}}\right]\{1234,12 \mid 34\} \\
& +\frac{n^{2}}{(n)_{4}}[\{1234,12 \mid 34\}+2 \operatorname{sim} .]-\frac{m^{2} n^{4}}{(m)_{2}(n)_{2}^{2}}\{12|34,12| 34\}
\end{aligned}
$$


iv. $\quad(1234,12|3| 4)=\frac{2 n^{2}}{(n)_{4}}\{1234,1234\}$

$$
\begin{aligned}
& -\left[\frac{m n^{3}}{(m)_{2}(n)_{2}^{2}}+\frac{n^{2}}{(n)_{3}}\right]\{1234,12 \mid 34\} \\
& -\frac{n^{2}}{(n)_{4}}[\{1234,12 \mid 34\}+2 \text { sim. }] \\
& \left.-\frac{m^{2} n^{2}}{(m)_{2}(n)_{3}}[1234,123 \mid 4\}+\{1234,124 \mid 3\}\right] \\
& +\frac{m^{3} n^{2}}{(m)_{3}(n)_{2}}\{1234,12|3| 4\}+\frac{m^{2} n^{3}}{(m)_{2}(n)_{2}^{2}}\{12|34,12| 34\} \\
& -\frac{m^{3} n^{2}}{(m)_{3}(n)_{2}}\{12|34,12| 3 \mid 4\}
\end{aligned}
$$

v. $\quad(1234,1|2| 3 \mid 4)=-\frac{6 n}{(n)_{4}}\{1234,1234\}$

$$
\begin{aligned}
& +\left[\frac{m n^{2}}{(m)_{2}(n)_{2}^{2}}+\frac{n^{2}}{(n)_{4}}\right][\{1234,12 \mid 34\}+2 \text { sim. }] \\
& +\frac{2 m^{2} n}{(m)_{2}(n)_{3}}[\{1234,123 \mid 4\}+3 \text { sim. }] \\
& -\frac{m^{3} n}{(m)_{3}(n)_{2}}[\{1234,12|3| 4\}+5 \text { sim. }] \\
& +\frac{m^{3}(m+1)}{(m)_{4}}\{1234,1|2| 3 \mid 4\} \\
& -\frac{m^{2} n^{2}}{(m)_{2}(n)_{2}^{2}}[\{12|34,12| 34\}+2 \text { sim. }] \\
& +\frac{m^{3} n}{(m)_{3}(n)_{2}}[\{12|34,12| 3 \mid 4\}+5 \text { sim. }] \\
& -\frac{m^{3}(m-1)}{(m)_{4}}[\{12|34,1| 2|3| 4\}+2 \text { sim. }]
\end{aligned}
$$

vi. $\quad \begin{aligned}(12|34,12| 34)= & -\frac{m n^{4}}{(m)_{2}(n)_{2}^{2}}\{1234,12 \mid 34\} \\ & +\frac{m^{2} n^{4}}{(m)_{2}(n)_{2}^{2}}\{12|34,12| 34\}\end{aligned}$

6.4. Three indices with the first nesting the second, which nests the third. We give a small selection of results when $m=4$. Note that $|\operatorname{Hom}(P, P(4))|=154$ when $P$ is the three-element chain. We assume $n_{1}=m, n_{2}=n$ and $n_{3}=p$. 
i. $\quad(1234,1234,1234)=\frac{p^{3}(p+1)}{(p)_{4}}\{1234,1234,1234\}$

$$
-\frac{p^{3}(p-1)}{(p)_{4}}[\{1234,1234,12 \mid 34\}+2 \operatorname{sim} .] \text {. }
$$

ii. $\quad(1234,1234,123 \mid 4)=-\frac{p^{2}(p+1)}{(p)_{4}}\{1234,1234,1234\}$

$$
\begin{aligned}
& +\frac{p^{2}(p-1)}{(p)_{4}}[\{1234,1234,12 \mid 34\}+2 \operatorname{sim} .] \\
& +\frac{n^{2} p^{3}}{(n)_{2}(p)_{3}}\{1234,1234,123 \mid 4\}
\end{aligned}
$$

iii. $\quad(1234,1234,12 \mid 34)=-\frac{p^{2}(p-1)}{(p)_{4}}\{1234,1234,1234\}$

$$
\begin{aligned}
& +\left[\frac{n p^{4}}{(n)_{2}(p)_{2}^{2}}+\frac{p^{3}}{(p)_{3}}\right]\{1234,1234,12 \mid 34\} \\
& +\frac{p^{2}}{(p)_{4}}[\{1234,1234,12 \mid 34\}+2 \text { sim. }] \\
& -\frac{n^{2} p^{4}}{(n)_{2}(p)_{2}^{2}}\{1234,12|34,12| 34\} .
\end{aligned}
$$

iv. $(1234,1234,12|3| 4)=\frac{2 p^{2}}{(p)_{4}}\{1234,1234,1234\}$

$$
\begin{aligned}
& -\frac{p^{2}}{(p)_{4}}[\{1234,1234,12 \mid 34\}+2 \text { sim. }] \\
& -\left[\frac{n p^{3}}{(n)_{2}(p)_{2}^{2}}+\frac{p^{3}}{(p)_{3}}\right]\{1234,1234,12 \mid 34\} \\
& -\frac{n^{2} p^{2}}{(n)_{2}(p)_{3}}[\{1234,1234,123 \mid 4\}+\{1234,1234,124 \mid 3\}] \\
& +\frac{n^{3} p^{2}}{(n)_{3}(p)_{2}}\{1234,1234,12|3| 4\} \\
& +\frac{n^{2} p^{3}}{(n)_{2}(p)_{2}^{2}}\{1234,12|34,12| 34\} \\
& -\frac{n^{3} p^{2}}{(n)_{3}(p)_{2}}\{1234,12|34,12| 3 \mid 4\}
\end{aligned}
$$


v. $(1234,1234,1|2| 3 \mid 4)=-\frac{6 p}{(p)_{4}}\{1234,1234,1234\}$

$$
\begin{aligned}
& +\left[\frac{n p^{2}}{(n)_{2}(p)_{2}^{2}}+\frac{p^{2}}{(p)_{4}}\right][\{1234,1234,12 \mid 34\}+2 \text { sim. }] \\
& +\frac{2 n^{2} p}{(n)_{2}(p)_{3}}[\{1234,1234,123 \mid 4\}+3 \text { sim. }] \\
& -\frac{n^{3} p}{(n)_{3}(p)_{2}}[\{1234,1234,12|3| 4\}+5 \text { sim. }] \\
& +\frac{n^{3}(n+1)}{(n)_{4}}\{1234,1234,1|2| 3 \mid 4\} \\
& -\frac{n^{2} p^{2}}{(n)_{2}(p)_{2}^{2}}[\{1234,12|34,12| 34\}+2 \text { sim. }] \\
& +\frac{n^{3} p}{(n)_{3}(p)_{2}}[\{1234,12|34,12| 3 \mid 4\}+5 \text { sim. }] \\
& -\frac{n^{3}(n-1)}{(n)_{4}}[\{1234,12|34,1| 2|3| 4\}+2 \text { sim. }] .
\end{aligned}
$$

vi. $\quad(1234,12|34,12| 34)=-\frac{n p^{4}}{(n)_{2}(p)_{2}^{2}}\{1234,1234,12 \mid 34\}$

$$
\begin{aligned}
& +\left[\frac{m}{(m)_{2}}+\frac{n^{2}}{(n)_{2}}\right] \frac{p^{4}}{(p)_{2}^{2}}\{1234,12|34,12| 34\} \\
& -\frac{m^{2} p^{4}}{(m)_{2}(p)_{2}^{2}}\{12|34,12| 34,12 \mid 34\} .
\end{aligned}
$$

vii. $(1234,12|34,12| 3 \mid 4)=\frac{n p^{3}}{(n)_{2}(p)_{2}^{2}}\{1234,1234,12 \mid 34\}$

$$
\begin{aligned}
& -\frac{n^{2} p^{2}}{(n)_{3}(p)_{2}}\{1234,1234,12|3| 4\} \\
& -\left[\frac{n^{2}}{(n)_{2}}+\frac{m}{(m)_{2}}\right] \frac{p^{3}}{(p)_{2}^{2}}\{1234,12|34,12| 34\} \\
& +\left[\frac{n^{2}}{(n)_{3}}+\frac{m^{2} n^{2}}{(m)_{2}(n)_{2}}\right] \frac{p^{2}}{(p)_{2}}\{1234,12|34,12| 3 \mid 4\} \\
& +\frac{m^{2} p^{3}}{(m)_{2}(p)_{2}^{2}}\{12|34,12| 34,12 \mid 34\} \\
& -\frac{m^{2} n^{2} p^{2}}{(m)_{2}(n)_{2}(p)_{2}}\{12|34,12| 34,12|3| 4\} .
\end{aligned}
$$


viii.

$$
\begin{aligned}
(1234,12|34,1| 2|3| 4)= & -\frac{n p^{2}}{(n)_{2}(p)_{2}^{2}}\{1234,1234,12 \mid 34\} \\
& +\frac{n^{2} p}{(n)_{3}(p)_{2}}[\{1234,1234,12|3| 4\}+\{1234,1234,1|2| 34\}] \\
& -\frac{n^{2}(n-1)}{(n)_{4}}\{1234,1234,1|2| 3 \mid 4\} \\
& +\left[\frac{m}{(m)_{2}}+\frac{n^{2}}{(n)_{2}}\right] \frac{p^{2}}{(p)_{2}^{2}}\{1234,12|34,12| 34\} \\
& -\left[\frac{n^{2}}{(n)_{2}}+\frac{n^{2}}{(n)_{3}}+\frac{m n^{2}}{(m)_{2}(n)_{2}}\right] \frac{p}{(p)_{2}}[\{1234,12|34,12| 3 \mid 4\} \\
& +\frac{n^{2}}{(n)_{4}}[\{1234,12|34,1| 2|3| 4\}+2 \text { sim. }] \\
& +\left[\frac{m n^{4}}{(m)_{2}(n)_{2}^{2}}+\frac{n^{3}}{(n)_{3}}\right]\{1234,12|34,1| 2|3| 4\} \\
& -\frac{m^{2} p^{2}}{(m)_{2}(p)_{2}^{2}}\{12|34,12| 34,12 \mid 34\} \\
& +\frac{m^{2} n^{2} p^{2}}{(m)_{2}(n)_{2}(p)_{2}}[\{12|34,12| 34,12|3| 4\} \\
& -\frac{m^{2} n^{4}}{(m)_{2}(n)_{2}^{2}}\{12|34,12| 34,1|2| 3 \mid 4\} . \\
& {[12|34,12| 34,1|2| 34\}] } \\
&
\end{aligned}
$$

ix. $\quad(12|34,12| 34,12 \mid 34)=-\frac{m p^{4}}{(m)_{2}(p)_{2}^{2}}\{1234,12|34,12| 34\}$

$$
+\frac{m^{2} p^{4}}{(m)_{2}(p)_{2}^{2}}\{12|34,12| 34,12 \mid 34\} \text {. }
$$

$\mathrm{X} . \quad(12|34,12| 34,12|3| 4)=\frac{m p^{3}}{(m)_{2}(p)_{2}^{2}}\{1234,12|34,12| 34\}$

$$
\begin{aligned}
& -\frac{m n^{2} p^{2}}{(m)_{2}(n)_{2}(p)_{2}}\{1234,12|34,12| 3 \mid 4\} \\
& -\frac{m^{2} p^{3}}{(m)_{2}(p)_{2}^{2}}\{12|34,12| 34,12 \mid 34\} \\
& +\frac{m^{2} n^{2} p^{2}}{(m)_{2}(n)_{2}(p)_{2}}\{12|34,12| 34,12|3| 4\} .
\end{aligned}
$$


xi. $\quad(12|34,12| 34,1|2| 3 \mid 4)=-\frac{m p^{2}}{(m)_{2}(p)_{2}^{2}}\{1234,12|34,12| 34\}$

$$
\begin{aligned}
& +\frac{m n^{2} p}{(m)_{2}(n)_{2}(p)_{2}}[\{1234,12|34,12| 3 \mid 4\} \\
& +\{1234,12|34,1| 2 \mid 34\}] \\
& -\frac{m n^{2}}{(m)_{2}(n)_{2}^{2}}\{1234,12|34,1| 2|3| 4\} \\
& +\frac{m^{2} p^{2}}{(m)_{2}(p)_{2}^{2}}\{12|34,12| 34,12 \mid 34\} \\
& -\frac{m^{2} n^{2} p}{(m)_{2}(n)_{2}(p)_{2}}[\{12|34,12| 34,12|3| 4\} \\
& \quad+\{12|34,12| 34,1|2| 34\}] \\
& +\frac{m^{2} n^{4}}{(m)_{2}(n)_{2}^{2}}\{12|34,12| 34,1|2| 3 \mid 4\} .
\end{aligned}
$$

\section{Appendix. Summary of definitions and notation used in this paper and its sequel}

Throughout $\mathbf{m}=\{1, \ldots, m\}$ and $\mathbf{n}_{p}=\left\{1, \ldots, n_{p}\right\}$ denote the sets consisting of the integers 1 to $m \geq 1$ and 1 to $n_{p} \geq 2$, respectively. $P$ always denotes the partially-ordered set, with elements $p, q, r$ etc., which defines the nesting relations on multiple indices $i=\left(i_{p}: p \in P\right)$, where for each $p \in P$ we have $i_{p} \in \mathbf{n}_{p}$.

Considerable use is made of arrays $\left(x_{i}\right),\left(y_{i}\right)$ etc. indexed by elements $i=$ $\left(i_{p}: p \in P\right)$ of the index set $\mathbf{I}=\prod\left\{\mathbf{n}_{p}: p \in P\right\}$, and when $|P|$ is 1,2 or 3 we usually denote the multiple indices $i_{1},\left(i_{1}, i_{2}\right)$ or $\left(i_{1}, i_{2}, i_{3}\right)$ by $i, i j$ or $i j k$ in the usual way.

Some use is made of the standard inner product defined as follows: if $x=\left(x_{i}\right)$, $y=\left(y_{i}\right),[x \mid y]=\sum_{i} x_{i} y_{i}$, the sum being over all $i \in \mathbf{I}$.

Little explicit mention will be made in this paper of the generalised wreath product group $G W(I)$ acting upon the elements of I so we will not repeat its definition here; see III and the references therein for futher details.

The lattice of all (set) partitions of the set $\mathbf{m}$ is denoted throughout by $P(\mathbf{m})$ with elements being written $(m=4) 1|2| 3|4,12| 3|4,123| 4$ etc. The lattice of all order-preserving maps from the partially-ordered set $P$ into $P(\mathbf{m})$ is denoted by $\operatorname{Hom}(P, P(\mathbf{m}))$ with typical elements being written abstractly as $\varphi=(\varphi(p): p \in$ $P)$ and concretely $(m=2)$ as $(12|34,12| 3 \mid 4)$ etc.

A number of functions and tensors are used in these papers which are labelled by elements $\varphi \in \operatorname{Hom}(P, P(\mathbf{m}))$. These include the generalised powers and 
incomplete factorials

$$
n^{\varphi}=\prod_{p \in P} n_{p}^{b(\varphi(p))}
$$

and

$$
(n)_{\varphi}=\prod_{p \in P}\left(n_{p}\right)_{(\varphi(p), \bigwedge\{\varphi(q): q>p\})}
$$

where

$$
\left(n_{p}\right)_{(\rho, \tau)}=\sum_{\pi} \mu(\rho, \pi)_{\zeta}(\pi, \tau) n_{p}^{\pi}
$$

$\rho, \tau \in\left(\mathbf{n}_{p}\right)$, this sum being over all $\pi \in P\left(\mathbf{n}_{p}\right)$, and the Möbius and zeta functions $\mu$ and $\varsigma$ being those of the lattice $P\left(\mathbf{n}_{p}\right)$. Here $n_{p}^{\pi}$ is just the integer $n_{p}$ raised to the power $b(\pi)$, where $b(\pi)$ denotes the number of blocks of the partition $\pi$.

The Möbius and zeta functions $\mu_{L}$ and $\zeta_{L}$ of any finite lattice (indeed any locally finite partially ordered set) $L$ are defined as follows:

$$
\varsigma_{L}(a, b)= \begin{cases}1 & \text { if } a \leq b \\ 0 & \text { otherwise }\end{cases}
$$

and $\mu_{L}(a, a)=1, \mu_{L}(a, c)=0$ if $a \not z c$, whilst for $a<c$ we have

$$
\mu_{L}(a, c)=-\sum_{a \leq b<c} \mu_{L}(a, b) .
$$

It is not hard to show that $\sum_{b} \zeta_{L}(a, b) \mu_{L}(b, c)=\sum_{b} \mu_{L}(a, b) \zeta_{L}(b, c)=\delta_{L}(a, c)$ which equals 1 if $a=c$, and equals 0 otherwise, the sums being over all $b \in L$. This is the principal property of $\mu_{L}$ used in the papers.

With this background we can recall Lemma 2.3 of III which states that $n^{\varphi}$ and $(n)_{\varphi}$ are Möbius inverses of one another: $n^{\varphi}=\sum \varsigma(\varphi, \psi)(n)_{\psi}$, and $(n)_{\varphi}=$ $\sum \mu(\varphi, \psi) n^{\psi}$, both sums being over all $\psi \in \operatorname{Hom}(P, P(\mathbf{m}))$.

We turn now to the tensors labelled by elements of $\operatorname{Hom}(P, P(\mathbf{m}))$, repeating definitions from III. Denote by $\delta^{i_{p}}$ the $n_{p} \times 1$ vector (that is, element of $R^{\mathbf{n}_{\mathbf{p}}}$ ) with values $\left(\delta^{i_{p}}\right)_{j_{p}}=1$ if $i_{p}=j_{p},=0$ otherwise, $i_{p}, j_{p} \in \mathbf{n}_{p}$. In terms of these vectors we then define the basis vectors $\left\{\delta^{i}: i \in \mathbf{I}\right\}$ by $\delta^{i}=\otimes_{p \in P} \delta^{i_{p}}, i_{p} \in \mathbf{n}_{p}$, $p \in P$. We also need to define the elements $\varphi^{h} \in \operatorname{Hom}(P, P(\mathbf{m}))$ derived from maps $h: \mathbf{m} \rightarrow \mathbf{I}=\prod\left(\mathbf{n}_{p}: p \in P\right)$ in terms of the kernels ker $h_{p}$ of the coordinate maps $h_{p}: \mathbf{m} \rightarrow \mathbf{n}_{p}$ by $\varphi^{h}(p)=\bigwedge\left\{\operatorname{ker} h_{q}: q>p\right\}$. Here ker $h_{p}$ is the partition of $\mathbf{m}$ which has $l_{1}$ and $l_{2}$ in the same block if and only if $h_{p}\left(l_{1}\right)=h_{p}\left(l_{2}\right)$. With these preliminaries we define the tensor

$$
\bar{h}=\delta^{h(1)} \otimes \cdots \otimes \delta^{h(m)}
$$

for any $h: \mathbf{m} \rightarrow \mathbf{I}$, and then for $\varphi \in \operatorname{Hom}(P, P(\mathbf{m}))$ we write

$$
A_{\varphi}=\sum_{\psi^{h}=\varphi} \bar{h}
$$


and

$$
R_{\varphi}=\sum_{\psi^{n} \geq \varphi} \bar{h}
$$

These tensors are abbreviated to $(n)_{\varphi}\langle\varphi\rangle$ and $[\varphi]$, and are called the association and relationship tensor, respectively. The corresponding generalised $k$-statistic tensor is $F_{\varphi}=\sum_{\psi} \mu(\psi, \varphi)(n)_{\psi}^{-1} A_{\psi}$, abbreviated $(\varphi)$. Transition matrices relating these three classes of tensors are given in Proposition 2.1 of III, the formulae being identical to those in Proposition 2.1 of III.

In order to define the generalised symmetric functions associated with the above tensors and labelled by elements $\varphi \in \operatorname{Hom}(P, P(\mathbf{m}))$ we need to refer to an array $x=\left(x_{i}(l): l \in \mathbf{m}, i \in \mathbf{I}\right)$ of indeterminates. We can also view $x$ as an array $x=\left(x_{i}: i \in \mathbf{I}\right)$ of $m$-tuple indeterminates labelled by $\mathbf{I}$, or as an array $(x(1), \ldots, x(m))$ of $\prod_{p \in P} n_{p}$-tuples (indexed lexicographically) labelled by $\mathbf{m}$. We temporarily adopt this second view and write $x=x(1) \otimes \cdots \otimes x(m)$. With this notation, and an extension of the inner product $[\cdot, \cdot]$ to tensor products via the formula $[\alpha \otimes \beta \mid a \otimes b]=[\alpha \mid a][\beta \mid b]$, we define the generalised symmetric functions $a_{\varphi}=\left[A_{\varphi} \mid x\right], s_{\varphi}=\left[R_{\varphi} \mid x\right]$ and $k_{\varphi}=\left[F_{\varphi} \mid x\right]$. At times we also write $k(\varphi), a(\varphi)$ or $k_{n_{1}, \ldots, n_{\bullet}}(\varphi)$, etc. where in the last mentioned example, the partially-ordered set $P$ is denoted by $\{1, \ldots, s\}$.

Finally, we recall that if $x$ now denotes an array of random variables whose joint distribution is invariant under the group $G W(\mathbf{I})$, then the generalised cumulants $f(\varphi)$ labelled by $\varphi \in \operatorname{Hom}(P, P(\mathbf{m}))$ are defined by $f(\varphi)=E\{k(\varphi)\}$, where $E$ denotes the expectation operator.

\section{References}

R. A. Bailey, C. E. Praeger, T. P. Speed and D. E. Taylor, Analysis of variance, forthcoming book.

E. J. Carney (1967) Computation of variances and covariances of variance component estimates, (PhD Thesis, Iowa State University).

E. J. Carney (1968) 'Relationship of generalized polykays to unrestricted sums for balanced complete finite populations', Ann. Math. Statist. 39, 634-656.

R. Hooke (1952) 'Some applications of bipolykays to the estimation of variance components and their moments', Ann. Math. Statist. 27, 80 98.

E. L. Kaplan (1952) 'Tensor notation and the sampling cumulants of $k$-statistics', Biometrika, 39, 319-323.

M. G. Kendall and A. Stuart (1969), The advanced theory of statistics, Volume 1. (Third Edition, Griffin, London).

T. P. Speed and R. A. Bailey (1982) 'On a class of association schemes derived from lattices of equivalence relations', Algebraic Structures and their Applications, edited by Philip Schultz, Cheryl E. Praeger and Robert P. Sullivan (Marcel Dekker, New York).

T. P. Speed (1984) 'On the Möbius function of $\operatorname{Hom}(P, Q)$ ', Bull. Austral. Math. Soc. 29, 39-46. 
T. P. Speed (1985) 'Dispersion models for factorial experiments'. Bull. International Statistical Institute. Proc. of 45th Session. 4, Amsterdam, 1985.

T. P. Speed (1986a) 'Cumulants and partition lattices II. generalised $k$-statistics', J. Austral. Math. Soc. Ser. A. 40, 34-53.

T. P. Speed (1986b) 'Cumulants and partition lattices III. multiply-indexed arrays', J. Austral. Math. Soc. Ser. A. 40, 161-182.

T. P. Speed (1986c) 'Anova models with random effects: An approach via symmetry', Essays in Time Series and Allied Processes: papers in honour of E. J. Hannan, Edited by J. Gani and M. B. Priestley, pp. 355-368 (Sheffeld: Applied Probability Trust).

Division of Mathematics and Statistics

CSIRO

Canberra 2601

Australia 